\title{
IMPACTO DOS ESQUEMAS PARAMETRIZAÇÃO CONVECTIVA DO MODELO WRF NA PREVISÃO DA PRECIPITAÇÃO, PREVISÃO DETERMINISTICA VS. PREVISÃO POR CONJUNTO
}

\author{
Lissette Guzmán (1), Vagner Anabor ${ }^{(2)}$, Franciano Puhales (3) \\ ${ }^{(1,2,3)}$ Grupo de Modelagem AtmosféricaUniversidade Federal de Santa Maria (GruMA-UFSM), Santa Maria, \\ Brazil, lissette19901109@gmail.com \\ (2) vanabor@gmail.com \\ (3) fpuhales@gmail.com
}

\section{Resumo:}

Na previsão numérica da precipitação, a resolução espacial do modelo é determinante na qualidade dos resultados. Para altas resoluções, ou seja, com espaçamento de grade inferior aos $4 \mathrm{~km}$, a convecção tem resolução explícita a partir da ativação dos esquemas de microf ísica. Com resoluções menores (espaçamento de grade maior de 12 $\mathrm{km}$ ) são empregados os esquemas de parametrização convectiva, também conhecidos como parametrizações implícitas, os quais parametrizam ou simplificam os processos que produzem a precipitação. Neste estudo foi realizado uma análise de sensibilidade do modelo WRF para 55 eventos de precipitação intensa na região sul do Brasil, perante nove esquemas de parametrização convectiva que integraram um sistema de previsão por conjunto (EPS), a partir do qual foi calculado o produto da precipitação média do conjunto (MPP). Considerando as observações de precipitação acumulada nas estações da Agencia Nacional das Àguas (ANA), foi avaliado o desempenho da previsão dos membros do EPS (EPSm) e o MPP, na previsão de limiares de precipitação de 1, 10, 25 e $50 \mathrm{~mm}$ em $24 \mathrm{~h}$. As previsões dos limiares de 1 e $10 \mathrm{~mm}$, obtidas pelos EPSm e o MPP, foram respectivamente os de maior índice de ameaça (TS), índice de sucesso (H) e menor false alarme (FAR), com os melhores resultados dos índices Hiedke (HSS) e de Clayton (CSS), sendo em geral super-prognosticados. Por sua parte, a previsão do limiar de $50 \mathrm{~mm}$ foi a de pior desempenho segundo os resultados do TS, H e o FAR, porém, foi o limiar com menor taxa de falsos alarmes (F) e, geralmente previsto um número de vezes similar ao observado. Com os esquemas de parametrização convectiva KainFritsch e Betts-Miller-Janjic foram obtidos bons resultados na previsão da precipitação moderada (limiares de 1 e $10 \mathrm{~mm}$ ), porém esses esquemas tiveram péssimo desempenho na previsão de precipitação intensa, especialmente superior a $50 \mathrm{~mm}$. O MPP poderia ser considerado como um bom preditor para valores não tão extremos de precipitação (excluindo $50 \mathrm{~mm}$ ) já que seu desempenho foi superior ao de vários dos EPSm. O CSS indicou que a previsão de ocorrência dos limiares obtida a partir desse produto, que foi efetivamente observada, resulta superior à da maioria das previsões determinísticas, reforçando a vantagem de utilizar o enfoque da previsão por conjuntos para obter prognosticos mais acurados da precipitação. A partir da analise dos resultados dos índices de desempenho, especialmente HSS e CSS, foi possível identificar aos membros com os esquemas de parametrização convectiva New Simplified Arakawa-Schubert e Tiedtke 
(membros 14 e 6) como os de melhores resultados na previsão de todos os limiares de precipitação analisados. Esses são esquema de fluxo de massa com componentes rasas (e profunda no caso de NSAS), e transporte de momentum, que a partir dos resultados deste trabalho são recomendados pelos autores para a obtenção de previsões determinísticas da precipitação na região sul do Brasil.

Palavras-chave: precipitação, parametrização convectiva, limiares, WRF, previsão por conjuntos.

\section{Introdução:}

Os eventos de intensas precipitações geram transtornos no desenvolvimento das atividades humanas, em especial quando esses elevados valores de precipitação que acontecem num período de tempo relativamente curto, abrangem grandes áreas de uma determinada geografia. Enxurradas, enchentes de rios, danos às culturas agrícolas, paralisação do transporte e de atividades comerciais, culturais e esportivas são algumas das consequências desses episódios extremos de chuva.

A região Sul do Brasil é especialmente propensa à ocorrência de grandes volumes de precipitação ao longo de todo o ano, seja pela passagens de frentes frias (Andrade, 2005) ou de sistemas convectivos de mesoescala (Anabor et al., 2008). Porém, não exite uma definição única do que constitui precipitação intensa. Muitas vezes a precipitação intensa é definida em termos de precipitação em uma única estação, outras, em termos da média sobre uma área determinada (Teixeira et Satyamurty, 2010). Enquanto alguns autores como Teixeira et Satyamurty (2007) empregam como critério limiares fixos de precipitação para definir-a como intensa (ex: mais de $50 \mathrm{~mm}$ em $24 \mathrm{~h}$ ), outros como Harnack et al. (1999), Junker et al. (1999) e Carvalho et al. (2002) utilizam como critério o da chuva exceder determinados percentis da climatologia mensual ou anual (ex: 20\%). Teixeira et Satyamurty (2010) definiram como precipitação intensa para região Sul do Brasil, aquela superior ao quantil $99 \%$ da climatologia mensual.

Embora os grandes avanços que tem sido registrados na previsão numérica do tempo ao longo das últimas décadas, na atualidade, a correta previsão espacial, temporal e quantitativa da precipitação ainda constitui um dos maiores desafios dos previsores do tempo. Erros nas observações fornecidas como condições iniciais aos modelos de previsão numérica, ou nas aproximações e parametrizações físicas utilizadas constituem os principais motivos de incerteza na hora de prever as precipitações (Fritsch et al., 1998).

Diante dessa realidade surge como alternativa a previsão por conjuntos, conceituada como uma ponderação entre os métodos estocásticos e dinâmicos (Epstein, 1969). Em um sistema de previsão por conjunto (Ensemble Prediction System, EPS) ao invés de executar o modelo uma única vez (previsão determinista), o modelo é executado várias vezes a partir de diferentes condições iniciais e/ou parametrizações físicas (EPS multifísica); ou podem ser usados vários modelos dentro do conjunto (EPS multi-modelo). A gama de soluções diferentes dos membros do conjunto, permitem uma medida objetiva da incerteza da previsão e também avaliar quão confiantes devemos estar em uma previsão determinista (Kalnay, 1987).

Toda uma gama de produtos básicos é produzida diretamente a partir de campos 
de saída dos EPS. A média do conjunto é uma média simples do valor da variável entre todos os membros do conjunto y geralmente obtêm melhores resultados que a previsão de controle na verificação das medidas padrões (erro médio absoluto, coeficiente de correlação de anomalias temporais, etc.) porque suaviza detalhes imprevisíveis e simplesmente apresenta os elementos mais previsíveis da previsão. Esse produto pode fornecer uma boa ideia do elemento da previsão que poderia ser previsto com confiança, mas não deve ser utilizado de forma individual, uma vez que raramente irá capturar o risco de eventos extremos.

O espalhamento do conjunto por sua parte, é calculado como o desvio padrão (nãotendencioso) da variável de saída do modelo, e fornece uma medida do nível de incerteza na previsão. Geralmete é plotado em gráficos sobreposto com a média do conjunto. Outros produtos são também utilizados, como a probabilidade básica, geralmente estimada como a simples proporção dos membros do conjunto que prevem a ocorrência de um evento em um local específico ou ponto de grade, além dos diagramas de espaguete, os mapas de todos os membros, as representações por quantis e os meteogramas (WMO, 2012).

Na previsão numérica da precipitação, a resolução espacial do modelo é determinante. Para altas resoluções, ou seja, com espaçamento de grade inferior aos $4 \mathrm{~km}$, a convecção tem resolução explícita a partir da ativação dos esquemas de microfísica. Com resoluções mais baixas (espaçamento de grade maior de $12 \mathrm{~km}$ ) são empregados os esquemas de parametrização convectiva, os quais parametrizam ou simplificam os processos que produzem a precipitação.

Ditos esquemas, conhecidos também como parametrizações implícitas, são os responsáveis pelos efeitos de escala sub-grade das nuvens convectivas e pretendem representar os fluxos verticais devido às correntes de ar ascendentes e descendentes, $\mathrm{e}$ os movimentos compensatórios fora das nuvens não resolvidos. Operam apenas em colunas individuais onde o esquema é ativado, fornecendo perfis verticais de aquecimento e umedecimento, tendências dos campos de nebulosidade e precipitação na coluna, porém, apenas alguns parametrizam a variação de momentum.

Algumas das parametrizações convectivas consideram tanto os efeitos da convecção rasa como os da convecção profunda, mas muitas estão focadas em reproduzir somente os efeitos isolados de uma ou outra sobre o ambiente. A maioria deles determinam um único resultado final do processo de convecção, enquanto outros adotam uma abordagem estocástica para determinar como a convecção influencia o ambiente ( Stensrud, 2009).

Mapes (1997) separou os esquema de convecção em dois tipos gerais com base na dimensão vertical do forçamento atmosférico que controla a convecção. Assim, esquemas de parametrização convectiva são agrupados em esquemas de controle de camada profunda (deep-layer control schemes, DLCS) e esquemas de controle de baixos níveis (low-level control schemes, LLCS). Os DLCS vinculam a criação de CAPE (convective available potential energy, energia potencial convectiva disponível) por processos de grande escala com o desenvolvimento da convecção, assumindo que a convecção consome imediatamente o CAPE produzido. Os LLCS amarram o desenvolvimento da 
convecção aos processos de iniciação convectiva nos quais o CIN (convective inhibition, inibição convectiva) é removido. Nestes esquemas o CAPE pode ser gerado e armazenado por longos períodos antes de ser consumido pelo sistema. Muitos esquemas incorporam elementos, tanto dos esquemas de controle da camada profunda como de controle de baixos níveis.

Segundo Stensrud (2009), os LLCS podem ser também chamados esquemas de controle de ativação, uma vez que assumem que a convecção profunda é determinada pelos processos físicos que controlam a iniciação convectiva. Esta suposição é coerente com a abordagem de previsão da convecção profunda baseada em ingredientes , na qual instabilidade, umidade e levantamento são necessários para gerar convecção profunda. Frequentemente são observadas amplas regiões nas quais o forçamento de grande escala gera CAPE (basicamente uma combinação de instabilidade e umidade em baixos níveis) ao longo de muitos dias, mas a convecção profunda só ocorre em áreas específicas, onde os forçamentos de mesoescala ou de pequena escala são capazes de movimentar as parcelas até o seu nível de convecção livre. Os LLCS tentam incorporar este tipo de comportamento ao funcionamento do sistema, preocupado-se com detalhes dos processos convectivos, e dos esquemas dinâmicos, sendo classificados como sistemas de fluxo de massa, uma vez que eles calculam a transferência de massa de um nível vertical para o nível seguinte.

Os DLCS muitas vezes assumem que o ambiente na grande escala fornece CAPE e que a convecção é um rápido e eficiente consumidor do CAPE produzido. Este tipo de esquema pode ser chamado de controle de equilíbrio, quase-equilíbrio, ou regime de ajuste do CAPE, uma vez que a convecção é assumida para manter a instabilidade do ambiente de grande escala em um estado de equilíbrio próximo da neutralidade.

O modelo Weather Research and Forecasting na versão 3.6 (WRF) de Skamarock et al. (2008b) possui diferentes opções de esquemas de parametrização convectiva, tanto do tipo DLCS, LLCS, como combinações delas. Na região sul do Brasil, composta pelos estados de Paraná, Santa Catarina e Rio Grande do Sul, não tem sido muito estudada a sensibilidade do modelo perante a variação desse tipo de parametrização, e se desconhece quais delas resultam mais adequadas para a previsão da precipitação, ou se por acaso, o enfoque da previsão por conjunto a traves dos produtos de EPS, resulta melhor.

O presente trabalho pretende avaliar o desempenho de nove diferentes esquemas de parametrização convectiva utilizados em um EPS, com vistas a identificar qual deles resulta mais adequado para a previsão de eventos de precipitação intensa na região sul do Brasil e, adicionalmente pretense-se comparar com o desempenho do produto da precipitação média do EPS.

\section{Materiais e Metodologia:}

As integrações do sistema de previsão por conjunto foram todas realizadas com o modelo WRF V.3.6. Todos os membros do conjunto tiveram igual configuração dos domínios e parametrizações físicas, diferenciando-se apenas pelos esquemas de parametrização convectiva utilizados.

Foram criados dois domínios, de 24 e 12 km de resolução horizontal, respectivamen- 
te, ambos com projeção geográfica Lambert (Fig. 1). O domínio exterior ou grade mãe, compreendido entre $16^{\circ} \mathrm{S}-38^{\circ} \mathrm{S}$ e $37^{\circ} \mathrm{O}-76^{\circ} \mathrm{O}$, com $143 \times 99$ pontos de grade, foi centrado sobre o território argentino, perto da fronteira com o Rio Grande do Sul. Um segundo domínio, estendendo-se entre $22^{\circ} \mathrm{S}-35^{\circ} \mathrm{S}$ e $44^{\circ} \mathrm{O}-61^{\circ} \mathrm{O}$, com $118 \times 136$ pontos de grade, centrado sobre o Rio Grande do Sul e abrangendo toda a região sul do Brasil, foi aninhado à grade mãe (aninhamento em duas vias). Ambos domínios foram estabelecidos com 27 níveis na vertical, e com saídas das simulações a cada $1 \mathrm{~h}$.

\section{WPS Domain Configuration}

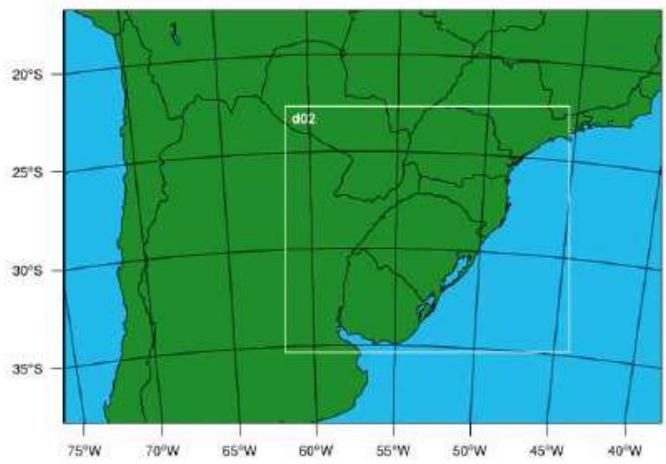

Fig. 1.- Domínios das simulações.

Empregaram-se condições iniciais e de contorno do NCEP FNL (Final) Operational Global Analysis, com uma resolução de $1^{\circ}$ e atualização das condições de contorno cada $6 \mathrm{~h}$. As parametrizações físicas comuns para todos os membros do EPS incluíram o esquema de microfísica de Lin (Lin et al., 1983), o qual considera processos de água na fase gelo e fase mista, resolvendo seis classes de hidrometeoros: vapor de água, água de nuvem, chuva, gelo de nuvem, neve e graupel. Utilizaram-se os esquemas RRTM (Mlayer et al., 1997) e Dudhia (Dudhia, 1989) para radiação de onda longa e curta, respetivamente, e Yonsei University (Hong et al., 2006) na parametrização de camada limite planetária.

Os nove esquemas de parametrização convectiva que diferenciaram as integrações do WRF para os diferentes membros do sistema de previsão por conjunto (EPSm), são sumarizados na Tabela 1.

\begin{tabular}{|clc|}
\hline Opcão & Parametrizacão & Referência \\
\hline 1 & Kain-Fritsch & Kain (2004) \\
\hline 2 & Betts-Miller-Janjic & Janjic (1994) \\
\hline 3 & Grell-Freitas Ensemble & Grell e Freitas (2014) \\
\hline 5 & Grell 3D & Grell (1993) Grell e Devenyi (2002) \\
\hline 6 & Tiedtke & Zhang et al. (2011) \\
\hline 14 & New Simplified Arakawa-Schubert & Han e Pan (2011) \\
\hline 4 & New Simplified Arakawa-Schubert (HWRF) & Han e Pan (2011) \\
\hline 93 & Grell-Devenyi (GD) Ensemble & Grell e Devenyi (2002) \\
\hline 99 & Old Kain-Fritsch & Kain e Fritsch (1990 \\
\hline
\end{tabular}

Tabela 1: Esquemas de parametrizações convectivas dos membros do EPS. 
A seguir são apresentadas algumas características dos esquemas de parametrização convectiva utilizados no EPS, descritas por Skamarock et al. (2008a).

Kain-Fritsch: Esquema sub-grade de convecção rasa e profunda, que utiliza uma aproximação de fluxo de massa com correntes descendentes, e uma escala temporal de remoção do CAPE (opção 1, m01).

Betts-Miller-Janjic: Esquema operacional do modelo Eta com ajuste da coluna úmida suavizado no sentido de um perfil bem misturado (opção 2, m02).

Grell-Devenyi (GD) Ensemble: Esquema multi-fechamento, multi-parâmetro, método conjunto com tipicamente 144 membros sub-grade (opção 93, m93).

Grell 3D: Versão melhorada do esquema GD que também pode ser utilizado em alta resolução (além de resoluções menores) se a opção de espalhamento da subsidência (cugd_avedx) está ativada (opção 5, m05).

Tiedtke: Esquema de tipo de fluxo de massa com escala temporal de remoção do CAPE, com componente superficial e transporte de momentum (opção 6, m06).

New Simplified Arakawa-Schubert: Novo esquema de fluxo de massa com componentes rasas e profunda, e transporte de momentum (opção 14, m14) .

New Simplified Arakawa-Schubert (HWRF): Novo esquema de fluxo de massa com componentes rasas e profunda, e transporte de momentum (opção 84, m84).

Grell-Freitas (GF): Esquema de GD melhorado que tenta suavizar a transição para escalas de resolução de nuvem (opção 3, m03).

Old Kain-Fritsch: Esquema de convecção profunda que utiliza uma abordagem de fluxo de massa com correntes descentes e escala temporal de remoção do CAPE (opção 99, m99).

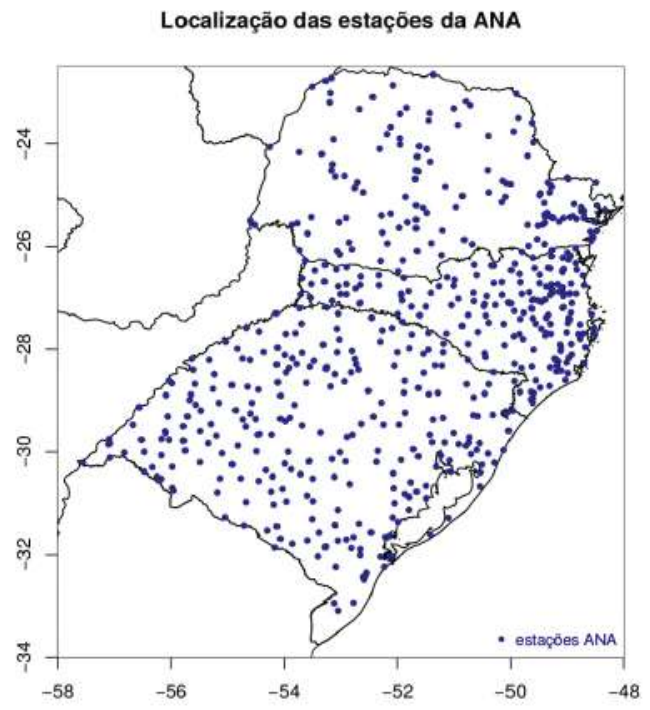

Os casos de estudos foram selecionados a partir dos dados das estações automáticas do Instituto Nacional de Meteorologia (INMET) no período 2008-2015, considerando apenas os dias que apresentaram acumulados de precipitação em $24 \mathrm{~h}$ (das 0 às 23 Z) superiores a $50 \mathrm{~mm}$, em pelo menos 10 estações da região de estudo. A partir de esse critério foi possível identificar 67 dias com essa cobertura espacial de precipitações intensas, 55 dos quais foram estudados neste trabalho.

As simulações dos casos abrangeram períodos de $48 \mathrm{~h}$,

Fig. 2.- Estações pluviométricas da ANA na região de estudo. 
sendo inicializadas às 12 UTC do dia anterior ao evento de precipitação intensa, para evitar a coincidência do tempo de spin-up do modelo e o período de observação dos eventos de precipitação intensa.

Para avaliar pontualmente os resultados das simulações, a comparação foi realizada com os dados de precipitação observada nas estações da ANA. Os valores de precipitação acumulada em $24 \mathrm{~h}$, medidos às $7 \mathrm{~h}$ local, estão

disponíveis no site HidroWeb da ANA. Contou-se com um total de 608 estações pluviométricas da ANA na região Sul do Brasil, sendo 277 no Rio Grande do Sul, 187 em Santa Catarina e 144 no Paraná (Fig. 2).

A avaliação das simulações do EPS foi realizada por meio de medidas ou índices de desempenho, conforme descrito por Wilks (2011) para previsões não-probabilísticas de eventos dicótomos. Ao comparar as simulações com as observações, pode acontecer uma de quatro situações, dependendo se o evento aconteceu (foi observado) ou não, e se foi previsto ou não. Convencionalmente, as quatro possibilidades são resumidas em tabelas de contingêncian 2x2 (como as da Tabela 2), sendo elas: acerto, falso alarme, evento não detectado ou rejeição correta, representadas respectivamente pelas letras $a$, $b, c, d$, sendo que $n=a+b+c+d$.

Previsão

$\operatorname{sim}$

não
Observacão

$\operatorname{sim}$

a

c não

b

d

Tabela 2- Tabela de contingência 2x2 (Adaptado de Wilks (2011)).

Como as observações da ANA são realizadas unicamente nos pontos com estações, ao utilizar as tabelas de contingência, o n ou tamanho da amostra, esteve dado pelo número de estações com reportes no período analisado, sendo avaliado o desempenho das simulações ou previsões somente nos pontos com estações da ANA com observações na data em questão.

Dessa forma, foram realizadas verificações das simulações do EPS por meio de índices de desempenho como taxa de acertos $(\mathrm{H})$, falsos alarmes (FAR e F), índice de sucesso crítico ou índice de ameaça (CSI ou TS) e a razão entre eventos estimados sim e observados sim (B). Também foi analisada a capacidade de acertos com a finalidade de verificar o desempenho das previsões, por meio dos índices de Hiedke (Hiedke Skill Score, HSS), Pierce (Pierce Skill Score, PSS) e Clayton (Clayton Skill Score, CSS) conforme Wilks (2011).

Ao ser coletadas às $7 \mathrm{~h}$ local, as observações da ANA, resultam comparáveis apenas com $24 \mathrm{~h}$, das $48 \mathrm{~h}$ simuladas com o WRF, sendo o mesmo das 21 às 45 h (09 UTC) no horário de verão da região sul do Brasil, e das 22 às 46 h (10 UTC) no horário normal. Para essa janela temporal foram calculados os valores de precipitação acumulada a partir das simulações e foi calculado o produto da precipitação média do EPS (mean precipitation product, MPP) (Equação 1). 


$$
M P P=\frac{1}{9} \sum_{i=1}^{9} P_{i}
$$

Conforme o anterior foram calculadas as medidas de desempenho para limiares de precipitação de 1, 10, 25 e 50 mm em 24 h, tanto para as simulações dos EPSm, como para o MMP.

\section{Resultados:}

O EPS foi utilizado na simulação dos 55 eventos de precipitação intensa na região sul do Brasil, para os quais foi avaliado o desempenho dos EPSm e do MPP, na previsão de quatro limiares de precipitação, a partir das observações da ANA. Seguidamente são

TS do EPS

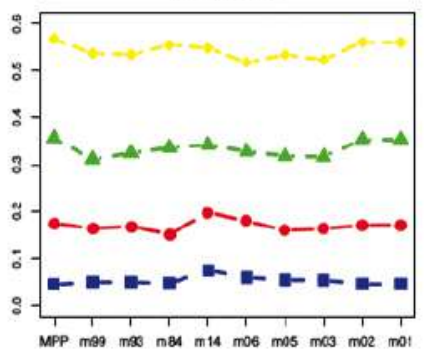

$-0-50 \mathrm{~mm} \rightarrow-25 \mathrm{~mm}^{*}+10 \mathrm{~mm}-1 \mathrm{~mm}$

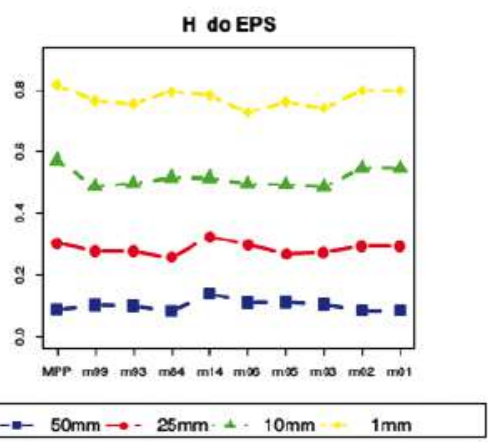
apresentados os valores médios das medidas calculadas para os casos de estudo.

Fig. 3.- TS dos EPSm e MPP nos pontos com observações da ANA.

De todas as previsões comparadas, os limiares mais baixos de precipitação obtiveram os melhores valores do TS (Fig. 3), que representa a razão entre a ocorrência prevista e o número de vezes que foi prevista e/ou observada, sendo desejados valores próximos à unidade. Tratando-se de limiares de pouca precipitação, o MPP resulta melhor que os EPSm, mas para as precipitações intensas os membros 14 e 6 (NSAS e Tiedtke) mostraram os melhores resultados.

Fig. 4.- H dos EPSm e MPP nos pontos com observações da ANA.

Os limiares de menor quantia de precipitação tiveram os melhores valores de $\mathrm{H}$ (Fig. 4), ou seja, a razão entre os acertos e o número de vezes que o evento ocorreu, foi mais próxima de 1. As previsões do MPP superaram aos EPSm na previsão de valores de 1 e $10 \mathrm{~mm}$ em $24 \mathrm{~h}$. Para acumulados de precipitação superiores a $25 \mathrm{~mm}$, novamente os membros 14 e 6 (NSAS e Tiedtke) foram os de melhores resultados.

Consequentemente, a previsão dos limiares de 50 e $25 \mathrm{~mm}$ apresentou a maior razão de falsos alarmes ou FAR (Fig. 5), sendo que para esses casos, o MPP superou os resultados de todos os EPSm. Tratando-se de limiares inferiores, os membros 14 e 6 (NSAS 

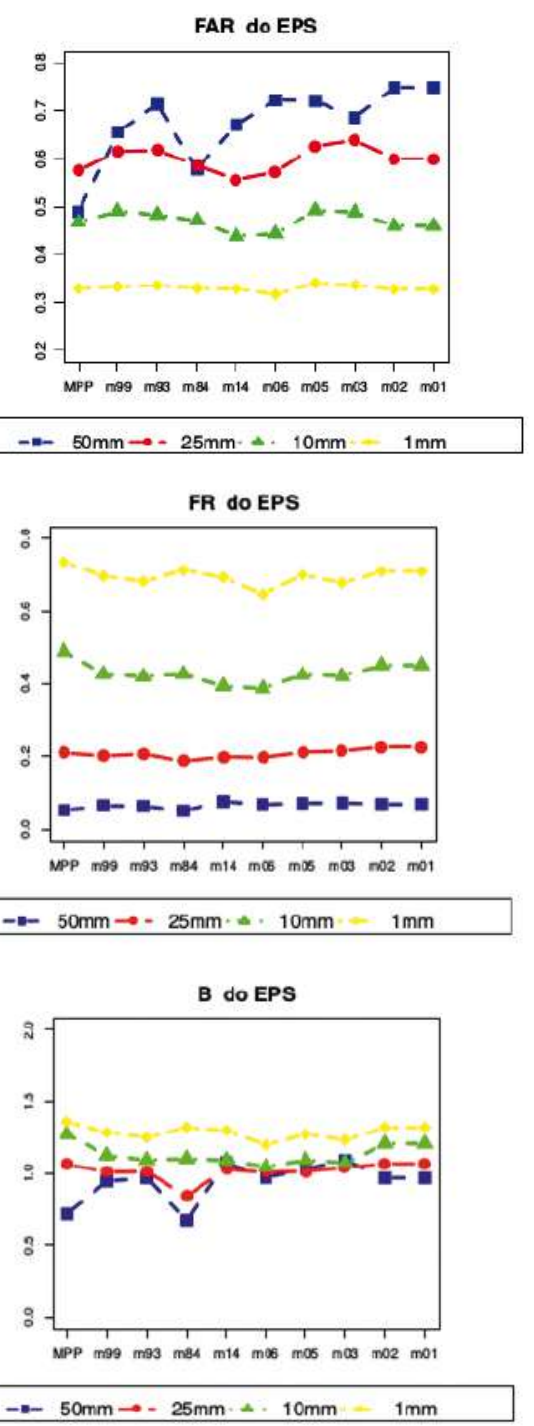

e Tiedtke) voltaram destacar-se positivamente. Porém, a precipitação intensa teve os melhores valores de F (Fig. 6), ou seja, a probabilidade de não detecção foi menor que para os outros limiares.

Fig. 5.- FAR dos EPSm e MPP nos pontos com observações da ANA.

Fig. 6.- F dos EPSm e MPP nos pontos com observações da ANA.

Em geral, os limiares de 25 e 50 $\mathrm{mm}$ de precipitação apresentaram os melhores valores de $\mathrm{B}$ (mais próximos de 1) mostrando que os eventos foram previstos aproximadamente o mesmo número de vezes que foram observados (Fig. 7), mas deve lembrar-se que $o$ B não fornece informação sobre a correspondência previsão observação de eventos particulares, pelo que não pode ser considerado como uma medida de precisão. Somente o membro 84 (NASA HWRF) e o MPP subprognosticaram esses limiares, como indicam os valores de $\mathrm{B}$ inferiores a 1. O B maior de 1 , apresentado pelos limiares de 1 e $10 \mathrm{~mm}$, confirma que esses eventos foram mais prognosticados do que observados.

Fig. 7.- B dos EPSm e MPP nos pontos com observações da ANA.

Como medida básica de precisão foi calculado o HSS, conhecido também como precisão de estimativa ao acaso. Previsões perfeitas recebem $H S S=1$, enquanto o valor zero indica que a previsão é equivalente a uma previsão de referência, e valores menores que zero, que as previsões são piores que a de referência. A previsão do limiar de $50 \mathrm{~mm}$ de precipitação obteve os resultados mais discretos do HSS (Fig. 8), com valores mais próximos do 0, e assim como para o limiar 
de $25 \mathrm{~mm}$, destacaram-se novamente os membros 14 e 6 (NSAS e Tiedtke) como os de melhor desempenho. Para esse último limiar os valores do índice foram em geral superiores, mas com grande variação entre os membros. Os limiares de 1 e $10 \mathrm{~mm}$ foram os de melhor desempenho e menor diferenciação entre os ESPm. Na previsão dos quatro limiares, o MPP apresentou valores que parecem refletir o caráter padronizador desse produto, melhorando o desempenho dos piores membros, mas ficando com rendimento inferior ao dos membros mais hábeis. Os resultados do HSS são coerentes com os obtidos por outros autores como Durai e Bhardwaj (2013) enquanto à maior acurácia da previsão numérica de valores moderados de precipitação e o grande grau de dificuldade ainda existente na previsão dos valores extremos dessa variável.
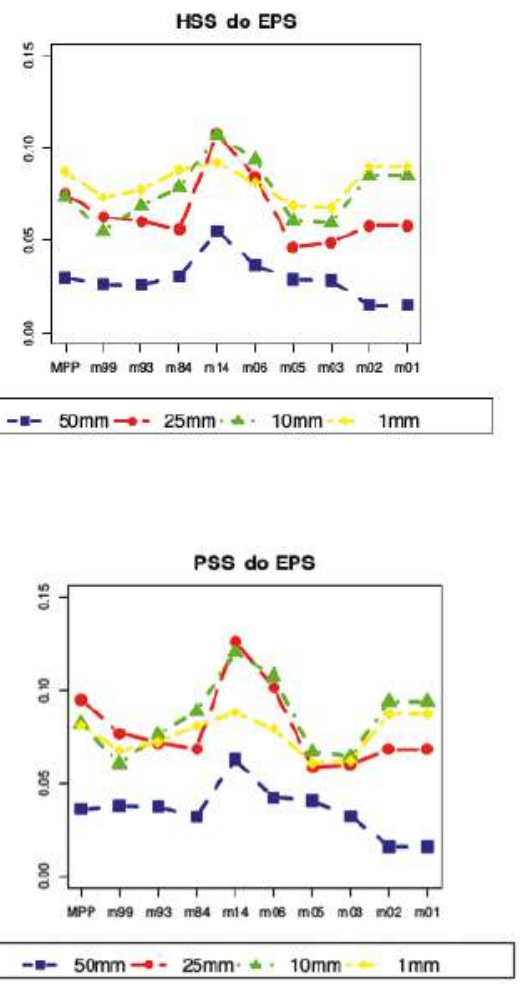

Fig 8.- HSS dos EPSm e MPP nos pontos com observações da ANA.

O PSS, similar à subtração entre $\mathrm{H}$ e F, teve resultados semelhantes aos do HSS, com valores do desempenho das previsões também discretos, mas sempre superiores aos de uma previsão aleatória (Fig. 9]). Resulta interessante o caso dos membros $1 \mathrm{e}$ 2 (Kain-Fritsch e Bets-Miller-Janjic), os quais tem valores razoavelmente bons para quase todos os limiares, mas possuim o pior rendimento no caso de limiar de $50 \mathrm{~mm}$. Esses dois esquemas parecem ser os piores na hora de prever a precipitação de mais de $50 \mathrm{~mm}$, enquanto que para o limiar de $25 \mathrm{~mm}$, os membros 3 e 5 (GrellFreitas e Grell-3D) foram os de menor desempenho.

Fig 9.- PSS dos EPSm e MPP nos pontos com observações da ANA.

O CSS indica habilidade positiva na medida em que o evento ocorre mais frequentemente quando previsto que quando não previsto. Previsões perfeitas apresentam $b=c=0$, e por tanto $C S S=1$, ao tempo que previsões aleatórias produzem $C S S=0$. Os valores do CSS na Fig. 10, mostram diferencias entre os diferentes esquemas analisados. Os membros 93 e 5 (GrellDevenyi-Ensemble e Grell-3D) destacam-se por apresentar valores desfavoráveis (mais próximos do 0) para os quatro limiares de precipitação analisados, enquanto o membro 
com o esquema 14 (NSAS) aparece como o de melhor desempenho geral. Os limiares de menor precipitação são os de melhores resultados, já os de 25 e $50 \mathrm{~mm}$ apresentam a maior variação ente os membros e desempenhos mais discretos. Os valores do CSS do MPP estiveram entre os maiores para os quatro limiares e não evidenciaram tanto o comportamento padronizador entre os EPSm, observado nos dois índices anteriores. Isso indica que o grau de acurácia das previsões do MPP resulta superior ao da maioria das previsões deterministas

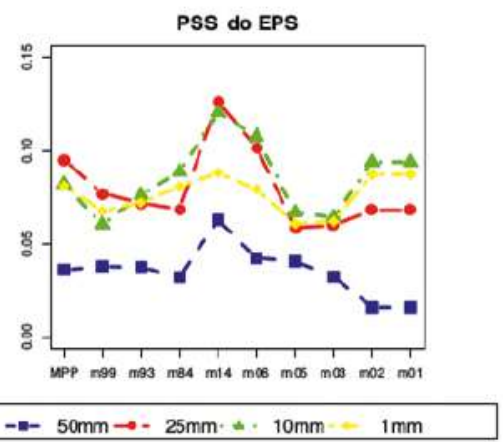
aqui analisadas, sendo os limiares de precipitação observados mais frequentemente quando previsto, que quando não previstos pelo MPP. O esquema 14 (NSAS) ratificou-se como a parametrização convectiva de melhor desempenho na previsão dos quatro limiares de precipitação considerados neste estudo.

Fig. 10.- CSS dos EPSm e MPP nos pontos com observações da ANA.

\section{Conclusões:}

Neste estudo foi realizado uma analise de sensibilidade do modelo WRF perante nove esquemas de parametrização convectiva para eventos de precipitação intensa na região sul do Brasil. As simulações correspondentes às nove configurações do modelo WRF integraram um EPS, a partir do qual foi obtido o MPP para cada caso de estudo.

Considerando as observações de precipitação acumulada da ANA, foi avaliado o desempenho da previsão dos EPSm e o MPP, na previsão de limiares de precipitação de 1, 10, 25 e $50 \mathrm{~mm}$ em $24 \mathrm{~h}$. Como esperado, as previsões dos limiares de 1 e $10 \mathrm{~mm}$, tanto pelos EPSm como pelo MPP, foram respectivamente os de maior TS, $\mathrm{H}$ e menor FAR e ratificado pelos resultados do HSS e do CSS, sendo esses limiares em geral superprognosticados. Por sua parte, a previsão do limiar de mais de $50 \mathrm{~mm}$ foi a de pior desempenho segundo os resultados do TS, H e o FAR, porém, foi também o limiar com menor taxa de falsos alarmes e, foi geralmente previsto um número de vezes similar ao observado.

Com os esquemas Kain-Fritsch e Betts-Miller-Janjic foram obtidos bons resultados na previsão da precipitação moderada (limiares de 1 e $10 \mathrm{~mm}$ ), porém esses esquemas tiveram péssimo desempenho na previsão de precipitação intensa, especialmente superior a $50 \mathrm{~mm}$. O MPP poderia ser considerado como um bom preditor para valores não tão extremos de precipitação (excluindo $50 \mathrm{~mm}$ ) já que seu desempenho foi superior ao de vários dos EPSm. O CSS indicou que a previsão de ocorrência dos limiares obtida a partir desse produto, que foi efetivamente observada, resulta superior à da maioria das previsões determinísticas, refoçando a vantagem de utilizar o enfoque da previsão por conjuntos para obter prognosticos mais acurados da precipitação. 
A partir da analise dos resultados dos índices de desempenho, especialmente HSS e CSS, foi possível identificar aos membros com os esquemas de parametrização convectiva New Simplified Arakawa-Schubert e Tiedtke (membros 14 e 6) como os de melhores resultados na previsão de qualquer um dos limiares de precipitação analisados. Esses são esquema de fluxo de massa com componentes rasas (e profunda no caso de NSAS), e transporte de momentum, que a partir dos resultados deste trabalho são recomendados pelos autores para a obtenção de previsões determinísticas da precipitação na região sul do Brasil, de não ser possível um enfoque estocástico.

\section{Referências}

- Anabor, V., Stensrud, D. J., Moraes, O. L. L. D., 2008: Serial upstream-propagating mesoscale convective system events over southeastern south america. Mon Wea Rev, 68(136), 3087-3105.

- Andrade, K. M. 2005: Climatologia e comportamento dos sistemas frontais sobre a América do sul. Mestrado em meteorologia, Instituto Nacional de Pesquisas Espaciais, São José dos Campos.

- Carvalho, L. M. V., Jones, C., Leibmann, B. 2002: Extreme precipitation events in southeastern South America and large-scale convective patterns in the south atlantic convergence zone.

- Dudhia, J. 1989: Numerical study of convection observed during the winter monsoon experiment using a mesoscale two-dimensional model. J Atmos Sci, 46, 3077-3107.

- Durai, V., Bhardwaj, R. 2013: Improving precipitation forecasts skill over india using a multimodel ensemble technique. Geofizika, 30, 120-140.

- Epstein, E. 1969: Stochastic dynamic predition.

- Fritsch, J., Houze, J. R., Adler, R., Bluestein, H., Bosart, L., Brown, J., Carr, F., Davis, C., Johnson, R., Junke, r. N., Kuo, Y. H., Rutledge, S., Smith, J., Toth, Z., Wilson, J., Zipse, r. E., Zrnic, D., 1998: Quantitative precipitation forecasting: Report of the eighth prospectus development team. Bull Am Meteorol Soc, 79, 285-299.

- Grell, G., 1993: Prognostic evaluation of assumptions used by cumulus parameterizations. Mon Wea Rev, 121, 764-787.

- Grell, G., Devenyi, D., 2002: A generalized approach to parameterizing convection combining ensemble and data assimilation techniques. Geophys Res Lett, 29, 1693.

- Grell, G., Freitas, S., 2014: A scale and aerosol aware stochastic convective parameterization for weather and air quality modeling. Atmos Chem Phys, 14, 5233-5250.

- Han, J., Pan, H., 2011: Revision of convection and vertical diffusion schemes in the ncep global forecast system. Atmos- Ocean, 26, 520-533.

- Harnack, R. P., Apel, K., Cermak, J. R., 1999: Heavy precipitation events in new jersey: Attendant upper-air conditions.

- Hong, S. Y., Noh, Y., Dudhia, J., 2006: A new vertical diffusion package with an explicit treatment of entrainment processes. Mon Wea Rev, 134, 2318-2341.

- Janjic, Z., 1994: The step-mountain eta coordinate model: Further developments of the convection, viscous sublayer, and turbulence closure schemes. Mon Wea Rev, 122, 927-945.

- Junker, N. W., Schneider, R. S., Fauver, S. L., 1999: A study of heavy rainfall events during the great midwest flood of 1993.

- Kain, J., 2004: The kain-fritsch convective parameterization: An update. J Appl Meteor, 43, 170-181.

- Kain, J., Fritsch, J., 1990: Ra one-dimensional entraining/detraining plume model and its application in convective parameterization. J Atmos Sci, 47, 2784-2802. 
- Kalnay, E. a. A., 1987: Forecasting forecast skill. Mon Wea Rev, 115, 349-356.

- Lin, Y. L., Farley, R., Orville, H., 1983: Bulk parameterization of the snow field in a cloud model. Jclimate Appl Met, 22, 1065-1092.

- Mapes, B., 1997: Equilibrium vs. activation control of large-scale variations of tropical deep convection in the physics and parameterization of moist atmospheric convection.

- Mlawer, E., Taubman, S., Brown, P., Iacono, M., Clough, S., 1997: Radiative transfer for inhomogeneous atmospheres: Rrtm, a validated correlated-k model for the longwave. J Geophys Res, 102, 16,663-16,682.

- SKAMAROCK, W., KLEMP, J., DUDHIA, J., GILL, D., BARKER, D., DUDA, M., HUANG, X., POWERS, J., WANG, W., 2008a: A description of the advanced research wrf version 3.

- SKAMAROCK, W., KLEMP, J., DUDHIA, J., GILL, D., BARKER, D., DUDA, M., HUANG, X., WANG, W., POWERS, J., 2008b: A description of the advanced research wrf version 3. URL http:// www2.mmm.ucar.edu/wrf/users/docs/arw_v3.pdf.

- STENSRUD, D., 2009: PARAMETERIZATION SCHEMES. Cambridge University Press, United States of America, New York.

- Teixeira, M., Satyamurty, P., 2010: Trends in the frequency of intense precipitation events in southern and southeastern brazil during 1960-2004. Journal of Climate, 24, 1913-1921.

- Teixeira, M. S., Satyamurty, P., 2007: Dynamical and synoptic characteristics of heavy rainfall episodes in southern brazil. Mon Wea Rev, 135, 598-617.

- Wilks, D., 2011: Statistical Methods in the Atmospheric Sciences. Academic Press, Oxford.

- World Meteorological Organization, 2012: Guidelines on ensemble prediction systems and forecasting. URL https://www.wmo.int/pages/prog/www/DPFS/Manual/documents/1091_ Guidelines_on_EPS_en.pdf, acesso em 14 set. 2015.

- Zhang, C., Wang, Y., Hamilton, K., 2011: Improved representation of boundary layer clouds over the southe-east pacific in arw-wrf using a modified tiedtke cumulus parameterization scheme. Mon Wea Rev, 139, 3489-3513. 